\title{
PREVENTING VIOLENCE AMONG ADOLESCENTS IN SCHOOL
}

\author{
Gergana Petrova, Elena Merdzhanova, Valentina Lalova \\ Department of Nursing, Faculty of Public Health, Medical University of Plovdiv, \\ Bulgaria.
}

\section{SUMMARY:}

Purpose: The present study aims to examine the violence among adolescents at school and outlines opportunities for preventing it.

Materials and Methods: The study was conducted at 17 schools on the territory of Plovdiv in two consecutive academic years: 2015/16 and 2016/ 17. Regular pupils are aged 11-14 years old. For the purpose of the study, have been requested on a regular basis for the number of regular pupils from 5th to 8th grade by grade and sex on the territory of the city of Plovdiv. 302 students were enrolled in the general study.

Results: More than half of 153 (55.6\%) of respondents said that they had suffered stress during the last year of their life, most often related to divorce of their parents, close-to-birth death, relative illness, relocation to another town, also moving to another school. These stressful situations in 162 $(58.9 \%)$ of teenagers are related to the school. There had been more of these situations for girls $83(63.4 \%)$ than for boys $79(54.9 \%)$. More than half of the students 158 (57.5\%) had been shown aggression or been witnessed. The results are the same for both sexes.

Conclusions: Girls have been objects of aggression and undergone more stress at school than boys. The main mechanisms for coping with stress and aggression in school are different in boys and girls. The boys said that they do it by themselves and part of the girls through a conversation with the family. A relatively small proportion has indicated the role of the school and school psychologist.

Keywords: Adolescents' health, adolescents in school, violence, stress.

\section{INTRODUCTION:}

Adolescent years have become a starting point for the formation of health behaviours that have lifetime consequences $[1,2]$. Adolescent age (adolescent, teenage) is a transition period from childhood to adulthood [3].

Despite the efforts of the government and the school as an institution, the question about school aggression still remains problematic, which, besides being very common, remains unrelated by the students on many occasions. Not a few children who have never managed to overcome such situations or they had, but in most cases alone or with the help of their family. The issue about the activity of school' psychologists and educators remains controversial, provided that aggression occurs mainly in the school environment.

\section{PURPOSE:}

The present study aims to examine the violence among adolescents at school and outlines opportunities for preventing it.

\section{MATERIALS AND METHODS:}

The study was conducted at 17 schools on the territory of Plovdiv in two consecutive academic years: 2015/16 and 2016/17. Schools were randomly selected from among all the schools with full-time education for pupils in 5th-8th grade on the territory of Plovdiv. Regular pupils are aged 1114 years old. For the purpose of the study, the Regional Inspectorate of Education and the Territorial Computing Center have been requested on a regular basis for the number of regular pupils from $5^{\text {th }}$ to $8^{\text {th }}$ grade by grade and sex on the territory of the city of Plovdiv. The youngsters included in the study were randomly selected through a statistical program. 302 students were enrolled in the general study. 27 of the latter were absent from school or did not fill in all the questions in the questionnaire on the day of the survey, which excluded them so that the results were based on 275 students.

The type of sociological survey is a direct group survey. A questionnaire containing 61 systematized questions (open, closed and mixed) was 
prepared for this purpose. Questions were grouped into four modules / panel: social demographic characteristics; physical activity and leisure time sports; eating habits; health factors such as smoking, alcohol use, various drugs, stress and aggression. Based on the purpose of the study, as well as on the volume and type of the data, the statistical methods used were: descriptive statistics and alternative analysis. The $\chi^{2}$ and Fisher's exact test criteria were applied.

\section{RESULTS:}

The subject of the survey were 275 students from the 5th, 6th, 7th and 8th grades of secondary schools in Plovdiv. Of these, the number of boys was $144(52.4 \% \pm 3.01)$ and girls $131(47.6 \% \pm$ $3.01)$. There was no association between the age and sex of the adolescents $(\mathrm{x} 2=5.979 ; \mathrm{df}=5 ; \mathrm{p}=$ 0.308 ). The highest number of students in the 5 th grade was $91(33.1 \% \pm 2.84)$, followed by students from the 6th grade $82(29.8 \% \pm 2.76), 7^{\text {th }}$ grade 55 $(20.0 \% \pm 2.41)$ and 8 th grade $47(17.1 \% \pm 2.27)$. A statistically significant difference in gender distribution in the different grades was not established (p>0.05). The relationship between the age and the place of residence of the students $(\mathrm{x} 2=15.84$; df $=5 ; \mathrm{p}=0.007)$ was established, but not between gender and place of residence ( $p>0.05)$. In every age, urban children were predominant, and that was a total of $249(90.5 \% \pm 1.76)$ of all. The largest proportion was of twelve-year-olds $\mathrm{n}=90$ and elevenyear-olds $n=83(32.7 \% \pm 2.83$ and $30.2 \% \pm 2.77)$.

The scientific study covers the group of socalled young adolescents or adolescents aged 1114. After analysis of the results, it was found that at the moment of completing the questionnaire there were children under 11 years of age $n=2$ $(0.7 \% \pm 0.51)$ and those who were 15 years old $n$ $=9(3.3 \% \pm 1.07)$.

More than half of $153(55.6 \%)$ of respondents said that they had suffered stress during the last year of their life, most often related to divorce of their parents, close-to-birth death, relative" illness, relocation to another town, also moving to another school. Those who have been survived stress are more girls $82(62.6 \%)$ than boys $71(49.3 \%),\left(\chi^{2}=\right.$ 4.909; $\mathbf{d f}=1 ; \mathbf{p}=\mathbf{0 . 0 2 9}$ ) (Figure 1$)$.

Fig. 1. Distribution of children, who had survived stressful situations during the last year, separated by gender

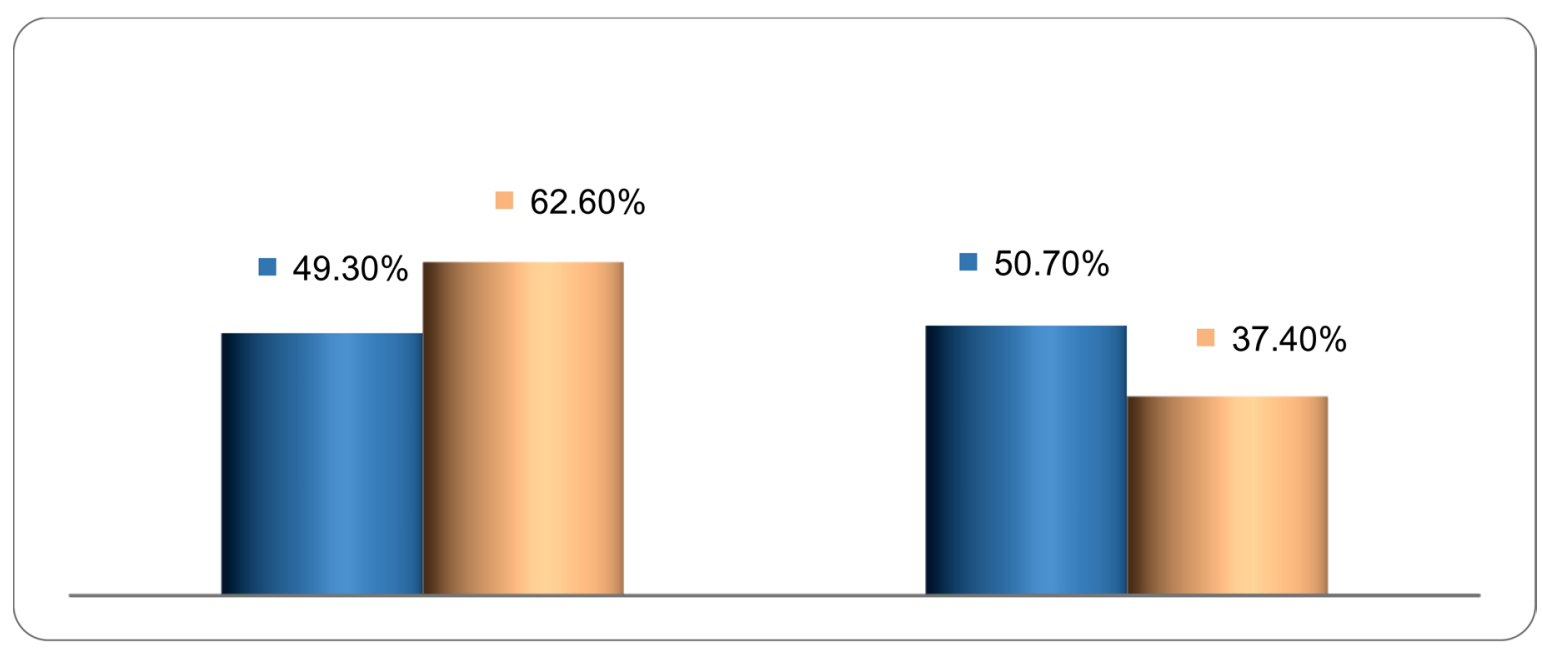

These stressful situations in $162(58.9 \%)$ of teenagers are related to the school. There had been more of these situations for girls $83(63.4 \%)$ than for boys $79(54.9 \%)$, gender did not have statistical significance $\left(\chi^{2}=\mathbf{2 . 0 4 6} ; \mathbf{d f}=\mathbf{1} ; \mathbf{p}=\mathbf{0 . 1 7 7}\right)($ Figure 2). 
Fig. 2. The relative share of stressful school-related situations

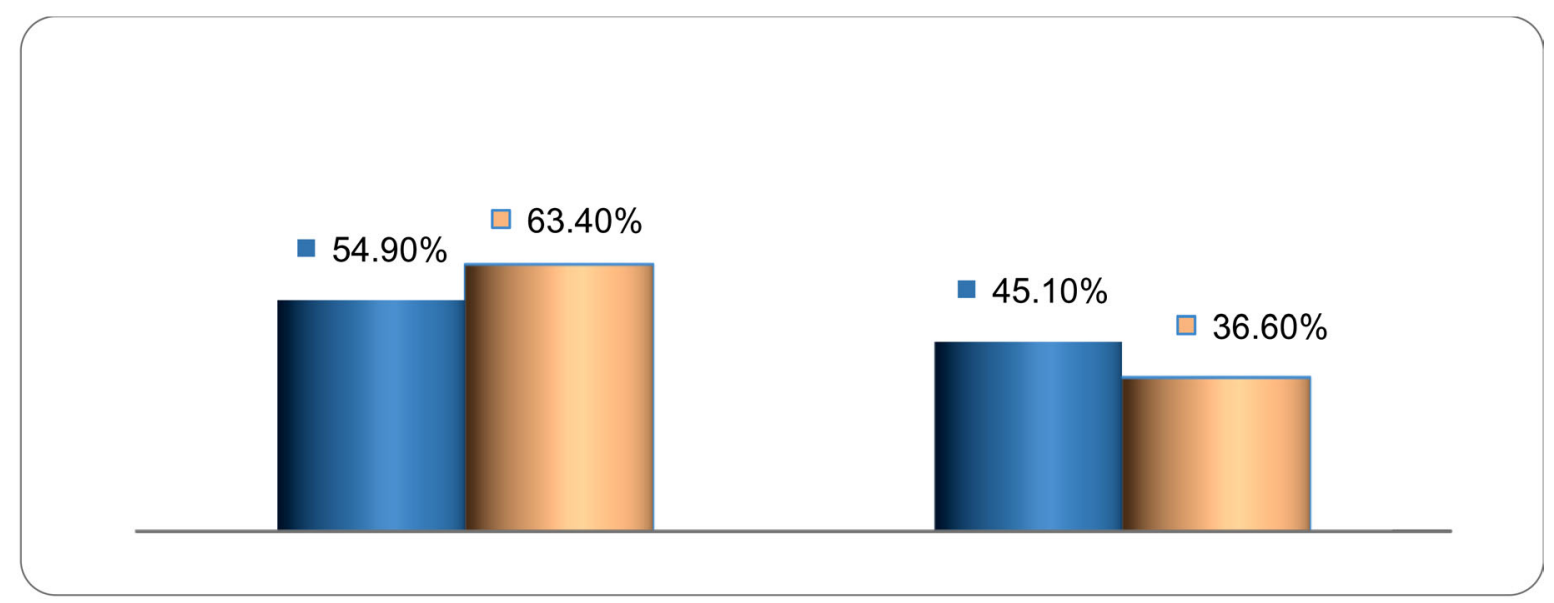

In $42(50 \%)$ of girls, this stress is related to the learning process, and in most of the boys 39 $(48.8 \%)$ it is associated with classmates of the same or another class, gender is not relevant to the nature of stress situations ( $p>0.05$ ).

More than half of the students $158(57.5 \%)$ had been shown aggression or been witnessed. The results are the same for both sexes. Under the aggression have been $93(33.8 \%)$ of all respondents, also relative shares roughly equal for both sexes. The girls are $46(35.1 \%)$ and the boys $47(32.6 \%)$
(Fig. 3). Gender does not affect ( $p>0.05$ ). Of those who have experienced stress or aggression in school, boys $14(9.9 \%)$ have not overcome this problem. The proportion of girls is $11(6.4 \%)$. It turns out that those who have dealt with the situation have been made it through conversations with family and friends as a very large share of boys 61 $(43.3 \%)$ and not a small part of girls $49(28.7 \%)$ have done it by themselves. Girls have not shared with anyone and have not looked for any assistance from family, friends, or school psychologist.

Fig. 3. How many young people have been objects of aggression by their classmates or others?

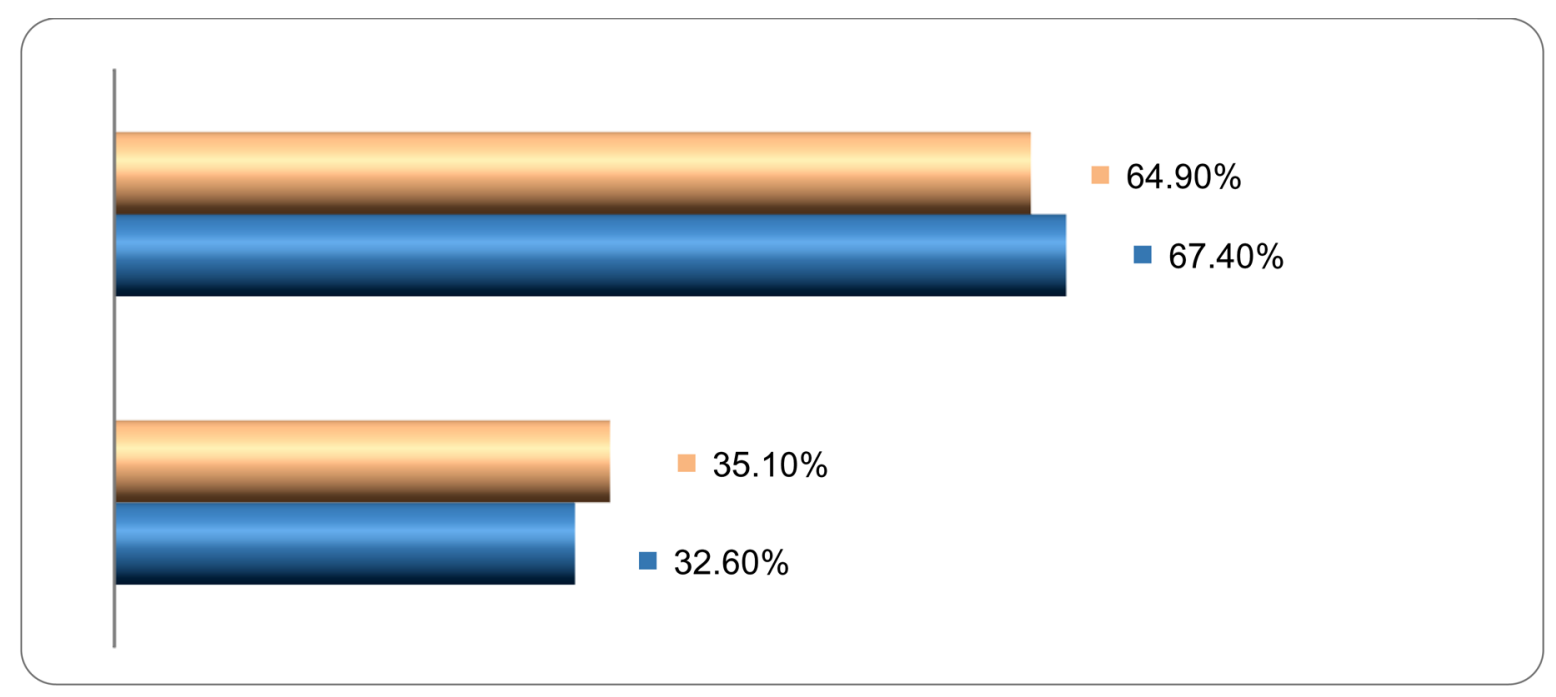

The content of health education is studied in several directions. One of them is related to the development of social skills and habits for healthy behavioural choices, including interpersonal relationships. The question remains open about the work of school psychologists and the school as an institution.

\section{DISCUSSION:}

A number of studies have shown an increase in aggression and harassment amongst children in recent years, most frequently being at their school age. This behaviour is very often associated with dislike of school as an institution and manifestation of unhealthy behaviour by aggressive children [4] 
Aggression among children and adolescents is getting alarmingly prevalent. It was showed that $29 \%$ and $10 \%$ of the students had moderate and high levels of aggression, respectively. Also, 30.6\% of them were moderate victims, and $45.6 \%$ were intense victims. The Social Cognitive Theory is the appropriate framework for the prediction of aggression behaviours in children and adolescents. Therefore, designing and implementing educational interventions based on this theory can help with the management of aggression in children and adolescents [5].

Healthy lifestyle habits during adolescence can prevent many of the diseases and disabilities in adulthood and later [6]. A healthy lifestyle is a system of activities consciously aimed at strengthening, preserving and maintaining the health of both the individual and society [7].

Every year, "The Movement - It's Health" Global Day is being held on May 10th. In the chronicle of World Health Days, celebrating this day begins in 2002. The World Day "Movement - It's Health" is linked to several main goals: integrating physical activity into everyday life; reducing through sport and physical activity the spread of risk factors for health - smoking, unhealthy eating, violence, stress and social exclusion [8].

Aggression and aggressive behaviour in children and adolescents can be related to physical and psychological health consequences, which continue in adulthood. Early programs for preventing violence in childhood and adolescence are designed to prevent or reduce aggressive behaviour in order to reduce the risk of short- and long-term complications [9]. As a result of the aggression manifested by some children, others had experienced stressful situations that were not always overcome. Very often, such cases remain unnoticed by parents, teachers, and even friends of the "victim." Often the consequences are for a life- time. For adolescents, self-determination and personality formation are forthcoming, as this is the period when the influence of family and friends is most important on them, even it is not consciously.

A major factor in the student" health culture, and in particular health awareness, is health enlightenment and health education in the school [10]. The content of health education is dealt with in several areas. One of them is related to the development of social skills and habits for healthy behaviour choices, including interpersonal relationships. The question remains about the work of school psychologists and the school as an institution.

\section{CONCLUSION:}

The study which had been conducted allows us to draw the following conclusions:

- Girls have been objects of aggression and undergone more stress at school than boys;

- The main mechanisms for coping with stress and aggression in school are different in boys and girls. The boys said that they do it by themselves and part of the girls through a conversation with the family. A relatively small proportion has indicated the role of the school and school psychologist.

\section{Conflicts of interest:}

There is no additional financing or conflict of interest at the realization if this scientific work. The scientific work has been presented to the Scientific Ethics Committee on September 21, 2017, which gave its opinion with an order of The Rector of Medical University - Plovdiv No. P-2550 / 12. 10. 2017. This scientific study has been conducted under the project NO-12/2019 of the Medical University of Plovdiv on the topic: Physical development and health behaviour of adolescents (11-14 years old) from different ethnic groups in the Municipality of Plovdiv.

\section{REFERENCES:}

1. Fernandez-Alvira JM, De Bourdeaudhuij I, Singh AS, Vik FN, Manios Y, Kovacs E, et al. Clustering of energy balance-related behaviors and parental education in European children: the ENERGY-project. Int J Behav Nutr Phys Act. 2013 Jan 15;10:5. [PubMed] [Crossref]

2. Jarvelaid M. Health damag- ing risk behaviours in adolescence [dissertation]. [Tartu (Es)]: University of Tartu. 2004. p. 153. [Internet].
3. Bobev
D, Genev
E.
[Crossref] [Pediatrics.] MI ARSO, Sofia. 2000. [in Bulgarian]

4. Schnohr C, Niclasen BV. Bullying among Greenlandic schoolchildren: development since 1994 and relations to health and health behaviour. Int $J$ Circumpolar Health. 2006 Sep; 65(4):305-12.

[PubMed]

5. Salimi N, Karimi-Shahanjarini A, Rezapur-Shahkolai F, Hamzeh B, Roshanaei G, Babamiri M. Aggression and its predictors among elementary stu- 
dents. J Inj Violence Res. 2019

Jul;11(2):159-170. [PubMed]

[Crossref]

6. Saffari M, Amini N, Eftekhar H, Ardebili H, Sanaeinasab M, Mahmoudi C, Piper N, Educational intervention on health related lifestyle changes among Iranian adolescents. Iran J Public Health. 2013; 42(2):
172-81.

7. Grancharova G, Velkova A, Aleksandrova S. Social Medicine. Pleven: Medical University Pleven, 2002. [In Bulgarian].

8. RHI. Movement is health. Yambol. 2008. [In Bulgarian].

9. Pawils S, Metzner F. [Violence prevention in childhood and adolescence-a brief over- view]. [in German] Bundesgesundheitsblatt Gesundheitsforschung Gesundheitsschutz. 2016 Jan;59(1):52-6. [PubMed] [Crossref]

10. Ivanov E. Students'health culture - factor for effective health promotion, Sofia: Gorex press, 2015. [In Bulgarian].

Please cite this article as: Petrova G, Merdzhanova E, Lalova V. Preventing violence among adolescents in school. $J$ of IMAB. 2020 Jan-Mar;26(1):2931-2935. DOI: https://doi.org/10.5272/jimab.2020261.2931

Received: 09/05/2019; Published online: 24/02/2020

Address for correspondence:

Assoc. Prof. Gergana Petrova, PhD

Department of Nursing, Faculty of Public Health, Medical University - Plovdiv 15, Vasil Aprilov blvd., 4002 Plovdiv, Bulgaria,

E-mail: gkpg21@yahoo.com 